\title{
THE NONEXISTENCE OF A CONTINUOUS SURJECTION FROM A CONTINUUM ONTO ITS SQUARE
}

\author{
HIDEFUMI KATSUURA
}

(Communicated by James E. West)

\begin{abstract}
In the late nineteenth century, the Italian mathematician Peano discovered a continuous surjection from $[0,1]$ onto $[0,1] \times[0,1]$. This led to the discovery, in the early twentieth century, of the Hahn-Mazurkiewicz Theorem, which states that a continuum (compact, connected metric space) is a continuous image of the unit interval $[0,1]$ if and only if it is locally connected. (Consequently, honoring Peano's discovery, we call a locally connected continuum a Peano continuum.) Combining this theorem and Urysohn's Lemma, one can prove the existence of a continuous surjection form a Peano continuum $X$ onto $X \times X$. This observation motivated the author to consider a continuous surjection from a continuum $X$ onto $X \times X$, and led to the discovery of a sufficient condition on a continuum for the nonexistence of such functions.
\end{abstract}

\section{INTRODUCTION}

In the late nineteenth century, the Italian mathematician Peano discovered a continuous surjection from $[0,1]$ onto $[0,1] \times[0,1]$. This led to the discovery, in the early twentieth century, of the Hahn-Mazurkiewicz Theorem which states that a continuum (compact, connected, metric space) is a continuous image of the unit interval $[0,1]$ if and only if it is locally connected. (Consequently, honoring Peano's discovery, we call a locally connected continuum a Peano continuum.) Combining this theorem and Urysohn's Lemma, one can prove the existence of a continuous surjection from a Peano continuum $X$ onto $X \times X$. This observation motivated the author to consider a continuous surjection from a continuum $X$ onto $X \times X$, and led to the discovery of a sufficient condition on a continuum for the nonexistence of such functions. Our main theorem is Theorem 14.

To begin with, we need some definitions and notation.

Definition 1. Throughout this paper, a space always means a compact metric space. Let $X$ be a space. A neighborhood of a point $p$ in $X$ is a subset that contains $p$ in its interior and itself may not be open. We say $X$ is connected im kleinen (locally connected) at $p$ if, for every open neighborhood $U$ of $p$,

Received by the editors February 9, 1989 and, in revised form, February 18, 1990.

1980 Mathematics Subject Classification (1985 Revision). Primary 54F20; Secondary 54C05. 
there exists a continuum neighborhood $W$ (respectively, an open, connected neighborhood $W$ ) of $p$ contained in $U$.

Even though the local connectedness of a space at a point implies connectedness im kleinen of the space at that point, the converse is false. We do, however, have the following:

Theorem 1. A space is locally connected (everywhere) if and only if it is connected im Kleinen (everywhere).

In other words, for most purpose, local connectedness and connectedness im Kleinen are the same. In particular, we may replace "locally connected" by "connected im kleinen" in the Hahn-Mazurkiewicz Theorem. It is the concept of connectedness im kleinen that we will generalize in order to state and prove our main theorem.

Definition 2. A space $X$ is $\sigma$-connected im kleinen at $p$ if for every open neighborhood $U$ of $p$ in $X$, there exists a closed neighborhood $W$ of $p$ contained in $U$ with countably many components.

Definition 3. In this paper the term ordinal number will mean "countable ordinal number" unless otherwise stated. The first infinite ordinal is denoted by $\omega$. Whenever an ordinal number is considered as a topological space, it always has the order topology. Recall that $0=\varnothing$, and if $\alpha$ is a nonzero ordinal, then $\alpha=\{\beta \mid \beta<\alpha\}$. For example, $\omega+1$ is homeomorphic to the harmonic sequence $\mathrm{Cl}\{1 / n \mid n=1,2,3, \ldots\}$ with the usual topology. (If $A$ is a subset of $X$, then $\mathrm{Cl} A$ will denote the closure of $A$ in $X$.)

Notation. The usual Cantor set is denoted by $S$ throughout this paper.

Definition 4. Let $X$ be a space. The quotient space obtained by identifying the points in $X \times\{1\}$ with a single point in the product space $X \times[0,1]$ is called the cone over $X$, and it is denoted by $C(X)$. (See Figure 1.) $X$ will be identified with the base $X \times\{0\}$ in $C(X)$.

Note that $C\left(\omega^{0}+1\right)$ is the only space in the above figures that is connected im kleinen. For each nonzero ordinal $\alpha, C\left(\omega^{\alpha}+1\right)$ is not connected im kleinen at $\omega^{\alpha}$, but it is $\sigma$-connected im kleinen everywhere. $C(S)$ is not $\sigma$-connected im kleinen at each point of $S$.

Our conjecture is the following:

Conjecture 1. If a continuum $X$ is not connected im kleinen at some point but is $\sigma$-connected im kleinen, then there is no continuous surjection from $X$ onto $X \times X$. 

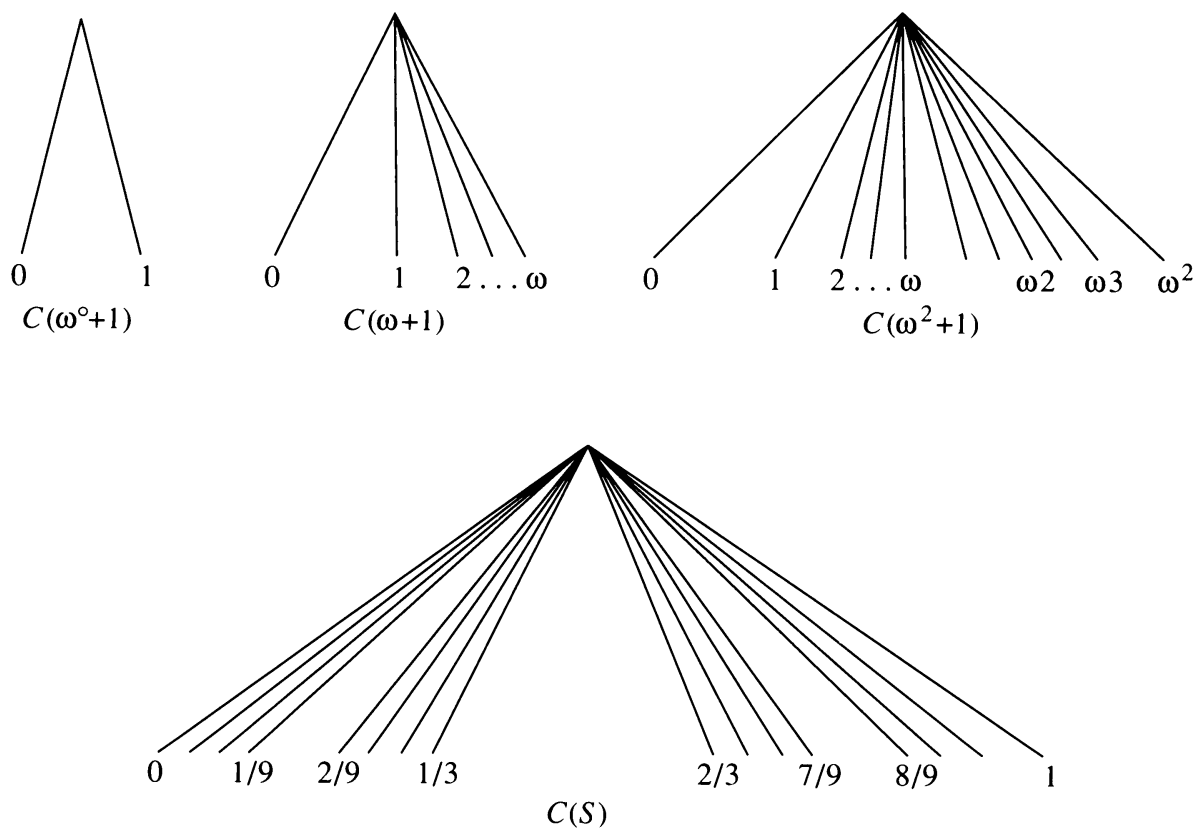

FIGURE 1

Unfortunately, we cannot prove the conjecture in full, but we will give a partial solution and show some of the difficulties in establishing it. An interesting consequence of our theorem is the following: If $\alpha$ is a nonzero ordinal, then there is no continuous surjection from $C\left(\omega^{\alpha}+1\right)$ onto $\left[C\left(\omega^{\alpha}+1\right)\right]^{2}$. However, there exists a continuous surjection from $C(S)$ onto $[C(S)]^{2}$. To construct such a function, note that every compact metric space is a continuous image of $S$ and $[C(S)]^{2}$ is contractible. For further interesting results about $C(S)$, see [1].

In order to state and prove our main theorem, we have to establish some unfamiliar definitions and related theorems, and the following two sections will be entirely devoted to these.

\section{THE $\alpha$-CONTINUUM}

Definition 5. Let $W$ be a closed subset of a space $X$. Let $W^{(0)}$ denote the quotient space of $W$ obtained by identifying each component with $W$ to a point. Let $\alpha$ be a nonzero ordinal. Suppose $W^{(\beta)}$ is defined for each $\beta<\alpha$.

(1) If $\alpha$ is not a limit ordinal, and if $\beta$ is the immediate predecessor of $\alpha$, then we define $W^{(\alpha)}$ to be the derived set of $W^{(\beta)}$.

(2) If $\alpha$ is a limit ordinal, then

$$
W^{(\alpha)}=\bigcap_{\beta<\alpha} W^{(\beta)}
$$


Therefore, by transfinite recursion, $W^{(\alpha)}$ is defined for each ordinal $\alpha . W$ is said to be $\alpha$-connected or an $\alpha$-continuum if $W^{(\alpha+1)}=\varnothing$, and least $\alpha$-connected or a least $\alpha$-continuum if $\alpha$ is the smallest ordinal such that $W^{(\alpha+1)}=\varnothing$.

Remarks [For the details, see Chapter 2 of 2]

(1) For each ordinal $\alpha,\left(\left(\omega^{\alpha}+1\right) \times[0,1]^{(0)}\right.$ and $(S \times[0,1])^{(0)}$ are homeomorphic to $\omega^{\alpha}+1$ and $S$, respectively.

(2) In the above definition, $W$ must be a closed set. For, otherwise, $W^{(0)}$ need not be a Hausdorff space.

(3) A continuum is always a 0 -continuum, but the converse is not necessarily true.

(4) If $\beta<\alpha$, then a $\beta$-continuum is an $\alpha$-continuum.

(5) $(\omega+1)^{(1)}=\{\omega\},(\omega+1)^{(2)}=\varnothing,\left(\omega^{2}+1\right)^{(1)}=\left\{\omega, \omega 2, \omega 3, \ldots, \omega^{2}\right\}$, $\left(\omega^{2}+1\right)^{(2)}=\left\{\omega^{2}\right\},\left(\omega^{2}+1\right)^{(3)}=\varnothing, \ldots$ In general, if $\alpha$ and $\beta$ are nonzero ordinals such that $\beta$ is less than or equal to $\alpha$, then we have $\left(\omega^{\alpha}+1\right)^{(\beta)}=\left\{\omega^{\beta} \cdot \mu \mid \mu\right.$ is an ordinal such that $\left.0<\omega^{\beta} \cdot \mu \leq \omega^{\alpha}\right\}$. In particular, if $\alpha$ is a nonzero ordinal, then $\left(\omega^{\alpha}+1\right)^{(\alpha)}=\left\{\omega^{\alpha}\right\}$ and $\left(\omega^{\alpha}+1\right)^{(\alpha+1)}=\varnothing$. Hence, for each ordinal $\alpha,\left(\omega^{\alpha}+1\right)$ is a least $\alpha$-continuum.

(6) If $X$ is a totally disconnected $\alpha$-continuum, and if $W$ is a closed subset of $X$, then $W$ is also an $\alpha$-continuum.

(7) A finite union of $\alpha$-continua is also an $\alpha$-continuum.

(8) Suppose $A$ is a closed subset of a space $X$, and $W$ is an $\alpha$-continuum in $X$ such that each component of $A$ meets $W$. Then $A \cup W$ is also an $\alpha$-continuum.

Definition 6. Let $\alpha$ be a nonzero ordinal. A predecessor sequence of $\alpha$ is a sequence $\left\langle\beta_{n}\right\rangle$ of ordinal numbers such that

(1) if $\alpha$ is not a limit ordinal, then $\beta_{n}=\alpha-1$ for every $n=1,2,3, \ldots$, and

(2) if $\alpha$ is a limit ordinal, then $\left\langle\beta_{n}\right\rangle$ is an increasing sequence of ordinal numbers less than $\alpha$ that converges to $\alpha$.

Theorem 2. Let $X$ and $Y$ be totally disconnected spaces and $f: X \rightarrow Y$ a continuous surjection. Suppose $b$ is in $Y^{(\alpha)}$ for some ordinal $\alpha$. Then $f^{-1}(b) \cap$ $X^{(\alpha)} \neq \varnothing$.

Proof. If $\alpha=0$, the result is clear. Let $\alpha>0$ be an ordinal number, and assume that, for every ordinal $\beta<\alpha$, the theorem holds.

Let $\left\langle\beta_{n}\right\rangle$ be a predecessor sequence of $\alpha$. Since $b$ is in $Y^{(\alpha)}$, there exists a sequence $\left\langle b_{n}\right\rangle$ of distinct points in $Y-\{b\}$ such that for every $n=$ $1,2,3, \ldots, b_{n}$ is in $Y^{(\beta n)}$ and $\left\langle b_{n}\right\rangle$ converges to $b$. By the transfinite induction hypothesis, there exists, for each $n=1,2,3, \ldots$, a point $a_{n}$ of $f^{-1}\left(b_{n}\right)$ such that $a_{n}$ is in $X^{(\beta n)}$. Since $X$ is compact, assume that the sequence $\left\langle a_{n}\right\rangle$ 
converges to some point $a$ in $X$. Since $f$ is continuous,

$$
f(a)=\lim _{n \rightarrow \infty} f\left(a_{n}\right)=\lim _{n \rightarrow \infty} b_{n}=b,
$$

which implies that $a$ is in $f^{-1}(b)$. Moreover, since $b_{n} \neq b$, we have $a_{n} \neq a$. Hence, $a$ is in $X^{(\alpha)}$.

Therefore, by transfinite induction, the theorem is proved.

Corollary 3. A continuous image of an $\alpha$-continuum is also an $\alpha$-continuum.

Remark. A compact space with countably many components can be shown to be an $\alpha$-continuum for some countable ordinal $\alpha$.

Next, we will investigate a product of $\alpha$-continua. But before we get into the discussion, we should keep in mind that the product of an $\alpha$-continuum and a $\beta$-continuum need not be an $(\alpha+\beta)$-continuum. One of the reasons for this is that we may have $(\alpha+\beta) \neq(\beta+\alpha)$.

Example 1. Let $X=\omega^{\omega+1}+1$. Then $X$ is a least $(\omega+1)$-continuum and $(\omega+1)+(\omega+1)=\omega 2+1$. However, $X^{2}=X \times X$ is not an $(\omega 2+1)-$ continuum. That $X \times X$ is a least $(\omega 2+2)$-continuum follows from Theorem 6 of this section.

This example suggests that we will need a new commutative addition $\oplus$ such that $(\omega+1) \oplus(\omega+1)=\omega 2+2$.

Definition 7. If $\zeta$ is a nonzero ordinal, and if $\zeta$ can be written in the form

$$
\zeta=\sum_{i=1}^{k} \omega^{\alpha_{i}} \cdot n_{i}
$$

where $\alpha_{1}>\alpha_{2}>\ldots>\alpha_{\kappa}$ are ordinals and $n_{i}$ is a nonzero natural number for each $i=1,2, \ldots, k$, then

$$
\sum_{i=1}^{k} \omega^{\alpha_{i}} \cdot n_{i}
$$

is said to be a standard representation of $\zeta$. The standard representation of 0 is $\omega^{0} 0$.

Theorem 4 [see Theorems 3-7 of 2]. Each countable ordinal number has a unique standard representation.

Definition 8. Let $\zeta=\sum_{i=1}^{k} \omega^{\alpha_{i}} \cdot n_{i}$ and $\delta=\sum_{j=1}^{h} \omega^{\beta_{j}} \cdot m_{j}$ be standard representations of ordinals $\zeta$ and $\delta$, respectively. Then $\zeta \oplus \delta$ is defined to be the sum

where

$$
\sum_{g=1}^{M} \omega^{\gamma_{g}} \cdot N_{g}
$$

(1) $\left\{\gamma_{g}\right\}_{g=1}^{M}=\left\{\alpha_{i}\right\}_{i=1}^{k} \cup\left\{\beta_{j}\right\}_{j=1}^{h}$, and $\gamma_{1}>\gamma_{2}>\ldots>\gamma_{M}$ (M is the number of elements in the set $\left\{\alpha_{i}\right\}_{i=1}^{k} \cup\left\{\beta_{j}\right\}_{j=1}^{h}$, and we are renaming each element of the set by $\gamma_{g}$ s in the order $\left.\gamma_{1}>\gamma_{2}>\ldots>\gamma_{M}\right)$, and 
(2) for each $g=1,2,3, \ldots, M, N_{g}$ is a natural number defined by

$$
N_{g}=\left\{\begin{array}{rr}
n_{i} & \text { if } \gamma_{g}=\alpha_{i} \text { for some } i=1,2, \ldots, k \\
& \text { but } \gamma_{g} \text { is not in }\left\{\beta_{j}: j=1,2, \ldots, h\right\}, \\
m_{j} & \text { if } \gamma_{g}=\beta_{j} \text { for some } j=1,2, \ldots, h \\
& \text { but } \gamma_{g} \text { is not in }\left\{\alpha_{i}: i=1,2, \ldots, k\right\}, \\
n_{i}+m_{j} & \text { if } \gamma_{g}=\alpha_{i} \text { for some } i=1,2, \ldots, k \\
& \text { and } \gamma_{g}=\beta_{j} \text { for some } j=1,2, \ldots, h .
\end{array}\right.
$$

Propositions. Let $\alpha, \beta$, and $\gamma$ be ordinals. The following seven propositions were proved in [2, pp. 19-22].

(1) $\alpha \oplus \beta=\beta \oplus \alpha$.

(2) If $\beta<\gamma$, then $\alpha \oplus \beta<\alpha \oplus \gamma$.

(3) If $\alpha \oplus \beta$ is a limit ordinal, then either $\alpha$ or $\beta$ is a limit ordinal.

(4) Let $\gamma$ be a limit ordinal. If $\left\langle\alpha_{n}\right\rangle$ and $\left\langle\beta_{n}\right\rangle$ are nondecreasing sequences of ordinals such that $\left\langle\alpha_{n} \oplus \beta_{n}\right\rangle$ is a predecessor sequence of $\gamma$, and if $\alpha_{n} \rightarrow \alpha$ and $\beta_{n} \rightarrow \beta$, then $\alpha \oplus \beta \geq \gamma$.

(5) If $\alpha \oplus \beta \geq \gamma$, then there exist ordinals $\alpha^{\prime}$ and $\beta^{\prime}$ such that $\alpha^{\prime} \oplus \beta^{\prime}=\gamma$, $\alpha^{\prime} \leq \alpha$ and $\beta^{\prime} \leq \beta$.

(6) Let $\gamma$ be a limit ordinal. If $\alpha \oplus \beta=\gamma$, then there exist nondecreasing sequences $\left\langle\alpha_{n}\right\rangle$ and $\left\langle\beta_{n}\right\rangle$ of ordinals such that

(i) $\alpha_{n} \rightarrow \alpha$ and $\beta_{n} \rightarrow \beta$,

(ii) either $\left\langle\alpha_{n}\right\rangle$ is a predecessor sequence of $\alpha$, or $\left\langle\beta_{n}\right\rangle$ is a predecessor sequence of $\beta$, and

(iii) $\left\langle\alpha_{n} \oplus \beta_{n}\right\rangle$ is a predecessor sequence of $\gamma$.

(7) Let $\gamma$ be a limit ordinal. If $\left\langle\alpha_{n} \oplus \beta_{n}\right\rangle$ is a predecessor sequence of $\gamma$, then it has a subsequence $\left\langle\alpha_{n i} \oplus \beta_{n i}\right\rangle$ such that

(i) $\left\langle\alpha_{n i} \oplus \beta_{n i}\right\rangle$ is a predecessor sequence of $\gamma$, and

(ii) $\left\langle\alpha_{n i}\right\rangle$ and $\left\langle\beta_{n i}\right\rangle$ are both nondecreasing.

Theorem 5. Let $\gamma$ be an ordinal. If $X$ and $Y$ are spaces, then

$$
(X \times Y)^{(\gamma)}=\bigcup\left\{X^{(\alpha)} \times Y^{(\beta)} \mid \alpha \oplus \beta=\gamma\right\} .
$$

(Note: For a given ordinal $\gamma$, there exist only a finitely many pairs of ordinals $\alpha$ and $\beta$ such that $\alpha \oplus \beta=\gamma$.)

Proof. Without loss of generality, assume that $X$ and $Y$ are both totally disconnected. If $\gamma=0$, then the theorem is clear.

Suppose that for some nonzero ordinal $\gamma$, the theorem is true for all ordinals 
$\mu<\gamma$. We will prove the theorem for $\gamma$. If $\gamma$ is not a limit ordinal, then

$$
\begin{aligned}
(X \times Y)^{(\gamma)} & =\left((X \times Y)^{(\gamma-1)}\right)^{(1)} \\
& =\bigcup\left\{X^{(\alpha)} \times Y^{(\beta)} \mid \alpha \oplus \beta=\gamma-1\right\}^{(1)} \text { by the inductive hypothesis } \\
& =\bigcup\left\{\left(X^{(\alpha+1)} \times Y^{(\beta)}\right) \cup\left(X^{(\alpha)} \times Y^{(\beta+1)}\right) \mid \alpha \oplus \beta=\gamma-1\right\} \\
& =\bigcup\left\{X^{(\alpha)} \times Y^{(\beta)} \mid \alpha \oplus \beta=\gamma\right\} .
\end{aligned}
$$

Next, suppose $\gamma$ is a limit ordinal. Let $\left\langle\gamma_{n}\right\rangle$ be a predecessor sequence of $\gamma$. Let $(p, q) \in(X \times Y)^{(\gamma)}$. Then there exists a sequence $\left\langle\left(p_{n}, q_{n}\right)\right\rangle$ of distinct points in $X \times Y-\{(p, q)\}$ such that $\left(p_{n}, q_{n}\right) \in(X \times Y)^{(\gamma n)}$ for every $n=1,2, \ldots$, and $\left(p_{n}, q_{n}\right) \rightarrow(p, q)$. Without loss of generality, assume that $\left\langle p_{n}\right\rangle$ is a sequence of distinct points in $X-\{p\}$. By the induction hypothesis, there are ordinals $\alpha_{n}$ and $\beta_{n}$ such that $\left(p_{n}, q_{n}\right) \in X^{(\alpha n)} \times Y^{(\beta n)}$ and $\alpha_{n} \oplus \beta_{n}=$ $\gamma_{n}$ for every $n=1,2,3, \ldots$. By Proposition 7 , we assume that $\left\langle\alpha_{n}\right\rangle$ and $\left\langle\beta_{n}\right\rangle$ are nondecreasing. Let $\alpha_{n} \rightarrow \alpha$ and $\beta_{n} \rightarrow \beta$. By Proposition 4, we have $\alpha \oplus \beta \geq \gamma$. Since $p_{n}$ is in $X-\{p\}$ for each $n=1,2, \ldots$, and $p_{n} \rightarrow p$, we have $p \in X^{(\alpha)}$. Since $q_{n} \in Y^{(\beta n)}$, we have $q \in Y^{(\beta)}$. Therefore, $(p, q) \in X^{(\alpha)} \times Y^{(\beta)}$. By Proposition 5, there exist ordinals $\varphi$ and $\lambda$ such that $\varphi \oplus \lambda=\gamma, \varphi \leq \alpha$, and $\lambda \leq \beta$.

Since $X^{(\alpha)} \times Y^{(\beta)} \subseteq X^{(\varphi)} \times Y^{(\lambda)} \subseteq \bigcup\left\{X^{(\varphi)} \times Y^{(\lambda)} \mid \varphi \oplus \lambda=\gamma\right\}$, we have

$$
(X \times Y)^{(\gamma)} \subseteq \bigcup\left\{X^{(\varphi)} \times Y^{(\lambda)} \mid \varphi \oplus \lambda=\gamma\right\}
$$

Conversely, suppose $(p, q) \in X^{(\alpha)} \times Y^{(\beta)}$, where $\alpha \oplus \beta=\gamma$. Then, by Proposition 6 , there exist nondecreasing sequences $\left\langle\alpha_{n}\right\rangle$ and $\left\langle\beta_{n}\right\rangle$ of ordinals such that

(i) $\alpha_{n} \rightarrow \alpha$ and $\beta_{n} \rightarrow \beta$,

(ii) either $\left\langle\alpha_{n}\right\rangle$ is a predecessor sequence of $\alpha$, or $\left\langle\beta_{n}\right\rangle$ is a predecessor of $\beta$, and

(iii) $\left\langle\alpha_{n} \oplus \beta_{n}\right\rangle$ is a predecessor sequence of $\gamma$.

Then there exists a sequence $\left\langle p_{n}\right\rangle$ of distinct points in $X-\{p\}$ that converges to $p$, and $p_{n}$ is in $X^{(\alpha n)}$ for every $n=1,2, \ldots$. Since $q$ is in $Y^{(\beta)}$, we know that $q$ is in $Y^{(\beta n)}$ for each $n=1,2, \ldots$ So $\left(p_{n}, q\right) \in X^{(\alpha n)} \times Y^{(\beta n)} \subset(X \times Y)^{(\gamma n)}$ for each $n$ by the inductive hypothesis. Moreover, $\left\langle\left(p_{n}, q\right)\right\rangle$ is a sequence of distinct points in $X \times Y-\{(p, q)\}$ converging to $(p, q)$ so $(p, q) \in(X \times Y)^{(\gamma)}$. Hence, we have proved that

$$
\bigcup\left\{X^{(\zeta)} \times Y^{(\delta)} \mid \zeta \oplus \delta=\gamma\right\} \subseteq(X \times Y)^{(\gamma)} .
$$

Therefore, by the transfinite induction, we have proved that

$$
(X \times Y)^{(\gamma)}=\bigcup\left\{X^{(\alpha)} \times Y^{(\beta)} \mid \alpha \oplus \beta=\gamma\right\}
$$

for each ordinal $\gamma$. 
Theorem 6. Let $\alpha$ and $\beta$ be ordinals. If $X$ is a least $\alpha$-continuum, and if $Y$ is a least $\beta$-continuum, then $X \times Y$ is a least $(\alpha \oplus \beta)$-continuum.

Proof. Let $\gamma=\alpha \oplus \beta$. Then

$$
(X \times Y)^{(\gamma)}=\bigcup\left\{X^{(\zeta)} \times Y^{(\delta)}: \zeta \oplus \delta=\gamma\right\} \supset X^{(\alpha)} \times Y^{(\beta)} \neq \varnothing .
$$

However, if $\zeta \oplus \delta=\gamma+1$, then $(\zeta>\alpha$ or $\delta>\beta)$, and $(\zeta>\beta$ or $\delta>\alpha)$. For otherwise, say $(\zeta>\alpha$ or $\delta>\beta)$ is false, we have $(\zeta \leq \alpha$ and $\delta \leq \beta)$, so that $\zeta \oplus \delta \leq \alpha \oplus \beta=\gamma$ by Proposition 2. This is a contradiction. Hence, if $\zeta \oplus \delta=\gamma+1$, then $X^{(\zeta)} \times Y^{(\delta)} \cup X^{(\delta)} \times Y^{(\zeta)}=\varnothing$. Thus $(X \times Y)^{(\gamma+1)}=$ $\bigcup\left\{X^{(\zeta)} \times Y^{(\delta)}: \zeta \oplus \delta=\gamma+1\right\}=\varnothing$.

Therefore, $X \times Y$ is a least $(\alpha \oplus \beta)$-continuum.

\section{3. $\alpha$-CONNECTEDNESS IM KLEINEN}

Definition 9. Let $\alpha$ be a nonzero ordinal. A space $X$ is said to be $\alpha$-connected im kleinen at $p \in X$ if for every open neighborhood $U$ of $p$, there exists a $\beta$ continuum neighborhood $W$ of $p$ contained in $U$ for some ordinal $\beta<\alpha . X$ is least $\alpha$-connected im kleinen if $X$ is $\alpha$-connected im kleinen everywhere, but for every ordinal $\gamma<\alpha$ there is a point $p \in X$ such that $X$ is not $\gamma$-connected im kleinen at $p$.

Remarks.

(1) A space $X$ is $\alpha$-connected im kleinen at $p \in X$ if for every open neighborhood $U$ of $p$, there exists a closed neighborhood $W$ of $p$ contained in $U$ such that $W^{(\alpha)}=\varnothing$.

(2) (connectedness im kleinen $)=(1$-connectedness im kleinen $)$.

(3) If $\alpha$ is a nonzero ordinal, then $C\left(\omega^{\alpha}+1\right)$ is not $\alpha$-connected im kleinen at $\omega^{\alpha}$, but it is least $(\alpha+1)$-connected im kleinen.

Example 2. In view of Remark (3), it is natural to ask: If $\alpha$ is a nonzero limit ordinal, is there a continuum that is least $\alpha$-connected im kleinen? The answer to this question is positive. We will give an example only for the case $\alpha=\omega$.

Let $A_{0}=\{0\}$, and let $A_{1}=\left\{1 / 2^{n} \mid n=1,2,3, \ldots\right\}$. Suppose $A_{n}$ is defined for some $n$. Then we define $A_{n+1}$ to be $A_{n+1}=\bigcup_{a \in A n}\left[\left(a^{\prime}-a\right) A_{1}+a\right]$, where for every $a$ in $A_{n}, a^{\prime}$ is the smallest element in $\left(\mathrm{Cl} A_{n}\right) \cup\{1\}$ such that $a^{\prime}>a$. For every $n=0,1,2,3, \ldots$, and for every $a$ in $A_{n}$, let $B_{n}(a)$ be the union of all line segments joining the point $\left(a, 1 / 2^{n}\right)$ to a point in $\left[\left(a^{\prime}-a\right) A_{1}+a\right] \times\left\{1 / 2^{n+1}\right\}$ in the $x y$-plane.

Let $Z=\operatorname{Cl}\left[\bigcup_{n=0}^{\infty} \bigcup_{a \in A_{n}} B_{n}(a)\right]$. See Figure 2.

Then, for every $n=1,2,3, \ldots, Z$ is not $n$-connected im kleinen at $\left(0,1 / 2^{n}\right)$, but it is least $\omega$-connected im kleinen. It is interesting to note that even though $\left(0,1 / 2^{n}\right) \rightarrow(0,0), Z$ is connected im kleinen at $(0,0)$. 


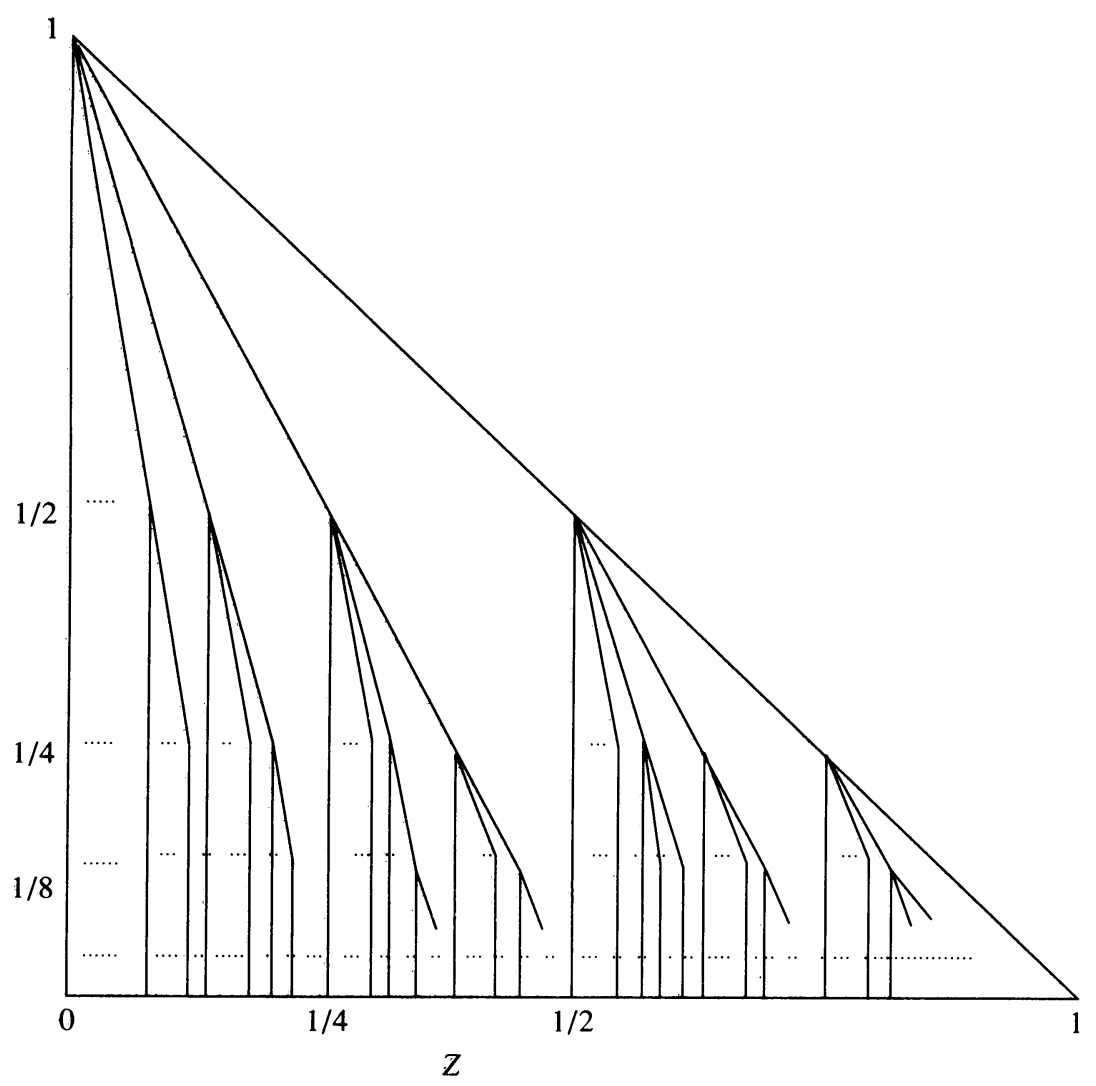

FIGURE 2

\section{Theorem 7}

(1) If a space $X$ is $\sigma$-connected im kleinen at $p$ in $X$, then $X$ is $\alpha$ connected im kleinen at $p$ for some ordinal $\alpha$.

(2) If a space is $\sigma$-connected im kleinen everywhere, then it is $\alpha$-connected im kleinen everywhere for some ordinal $\alpha$.

Idea of the proof: We simply note that $X$ has a countable open base, and if $\left\{\alpha_{n} \mid n=1,2, \ldots\right\}$ is a set of countable ordinals, then it has an upper bound that is also a countable ordinal.

Corollary 8. If a space is $\sigma$-connected im kleinen everywhere, then it is least $\alpha$-connected im kleinen everywhere for some ordinal $\alpha$.

Notation. If $X$ is a space, and if $\alpha$ is a nonzero ordinal, then let

$$
\Omega_{\alpha}(X)=\operatorname{Cl}\{p \in X \mid X \text { is not a } \alpha \text {-connected im kleinen at } p\} \text {. }
$$

Theorem 9. If $f: X \rightarrow Y$ is a continuous surjection, then for each nonzero ordinal $\alpha$, we have

$$
f\left(\Omega_{\alpha}(X)\right) \supset \Omega_{\alpha}(Y) .
$$


Proof. Let $p \in Y$ be a point at which $Y$ is not $\alpha$-connected im kleinen. Then there exists an open neighborhood $U$ of $p$ such that, if $W$ is a closed neighborhood of $p$ contained in $U, W^{(\alpha)} \neq \varnothing$. Suppose for every $x \in f^{-1}(p)$, $X$ is $\alpha$-connected im kleinen at $x$. Then for every $x \in f^{-1}(p)$, there exists a closed neighborhood $W(x)$ of $x$ contained in $f^{-1}(U)$ such that $W(x)^{(\alpha)}=\varnothing$. Since $f^{-1}(p)$ is compact, there exists a finite set of points $\left\{x_{1}, x_{2}, \ldots, x_{n}\right\}$ in $f^{-1}(p)$ such that $f^{-1}(p) \subset \bigcup\left\{\operatorname{Int} W\left(x_{i}\right) \mid i=1,2, \ldots, n\right\}$. By compactness of $X$ and continuity of $f$, we have

$$
p \in \operatorname{Int} f\left(\bigcup_{i=1}^{n} W\left(x_{i}\right)\right),
$$

so that $f\left(\bigcup_{i=1}^{n} W\left(x_{i}\right)\right)$ is a closed neighborhood of $p$ contained in $U$. But $\left[f\left(\bigcup_{i=1}^{n} W\left(x_{i}\right)\right)\right]^{(\alpha)}=\varnothing$ by Corollary 3 . This is a contradiction.

Therefore, there exists a point $x$ in $f^{-1}(p)$ at which $X$ is not $\alpha$-connected im kleinen. Hence

$$
\begin{aligned}
& f(\{x \in X \mid X \text { is not } \alpha \text {-connected im kleinen at } x\}) \\
& \qquad\{y \in Y \mid Y \text { is not } \alpha \text {-connected im kleinen at } y\} .
\end{aligned}
$$

By the continuity of $f$, we have $f\left(\Omega_{\alpha}(X)\right) \supset \Omega_{\alpha}(Y)$.

Corollary 10. Let $\alpha$ be a nonzero ordinal. A continuous image of a continuum that is $\alpha$-connected im kleinen ( $\sigma$-connected im kleinen) is also $\alpha$-connected im kleinen ( $\sigma$-connected im kleinen, respectively).

Notation. If $A$ and $W$ are subsets of a space, then we denote by $H(A, W)$ the following set:

$$
\bigcup\{K \mid K \text { is a component of } W \text { that meets the closure of } A\} .
$$

Hence, if $W$ is closed, then so is $H(A, W)$.

Theorem 11 [(see Theorems 6-12 of 2]. Let $\alpha$ be a nonzero ordinal. Then a space $X$ is $\alpha$-connected im kleinen at $p$ in $X$ if and only if, for every closed neighborhood $W$ of $p$, there exists an open neighborhood $O$ of $p$ such that $H(O, W)^{(\alpha)}=\varnothing$.

Proof. Suppose $X$ is $\alpha$-connected im kleinen at $p$ in $X$. Let $W$ be a closed neighborhood of $p$. Then, for some ordinal $\beta<\alpha$, there exists a $\beta$-continuum neighborhood $V$ of $p$ that is contained in Int $W$. Since every component of $H(V, W)$ meets $V$, and, since $V \cup H(V, W)=H(V, W), H(V, W)$ must be a $\beta$-continuum. But then, for every open neighborhood $O$ of $p$ contained in Int $V, H(O, W)$ is a $\beta$-continuum so that $H(O, W)^{(\alpha)}=\varnothing$.

Conversely, suppose that for every closed neighborhood $W$ of $p$, there exists an open neighborhood $O$ of $p$ such that $H(O, W)^{(\alpha)}=\varnothing$. Let $U$ be an open neighborhood of $p$. Let $W$ be a closed neighborhood of $p$ in $U$. Then, by our assumption, there exists an open neighborhood $V$ of $p$ such that 
$H(V, W)^{(\alpha)}=\varnothing$. Moreover, $p \in V \cap \operatorname{Int} W \subset \operatorname{Int} H(V, W) \subset H(V, W) \subset U$. Hence, $X$ is $\alpha$-connected im kleinen at $p$.

Corollary 12. Let $\alpha$ be a nonzero ordinal. Then a space $X$ is not $\alpha$-connected im kleinen at $p$ in $X$ if and only if there exists a closed neighborhood $W$ of $p$ such that, if $O$ is an open neighborhood of $p$, then $H(O, W)^{(\alpha)} \neq \varnothing$.

Definiiton 11. A nonzero countable ordinal is said to be of type 1 if it has a standard representation of the following form:

$$
\sum_{i=1}^{k} \omega^{\alpha_{i}}, \text { for some nonzero ordinals } \alpha_{1}>\alpha_{2}>\ldots>\alpha_{k} .
$$

Type 1 ordinals are necessarily limit ordinals. All other countable ordinals are said to be of type 2 . All nonlimit ordinals are of type 2 .

Our main reason for separating countable ordinals into these two types is that if $\alpha$ is an ordinal of type 1 , then there is no ordinal $\beta<\alpha$ such that $\beta \oplus \beta \geq \alpha$, while if $\alpha \geq 2$ is a type 2 ordinal, then there always exists an ordinal $\beta<\alpha$ such that $\beta \oplus \beta \geq \alpha$.

Theorem 13. Let $\alpha \geq \beta$ be nonzero ordinals, and let $X$ and $Y$ be continua that are least $\alpha$-connected im kleinen and $\beta$-connected im kleinen, respectively.

(1) If $\alpha$ is a type 1 ordinal, then $X \times Y$ is least $\alpha$-connected im kleinen.

(2) If $\alpha \geq 2$ is a type 2 ordinal, then $X \times X$ is not $\alpha$-connected im kleinen. Proof.

(1) Let $(p, q) \in X \times Y$, and $U \times V$ be an open neighborhood of $(p, q)$ in $X \times Y$. Then for some ordinals $\zeta<\alpha$ and $\delta<\beta$, there exists a $\zeta$-continuum neighborhood $A$ of $p$ in $U$ and a $\delta$-continuum neighborhood $B$ of $q$ in $V$. But, by Theorem $6, A \times B$ is a $(\zeta \oplus \delta)$-continuum neighborhood of $(p, q)$ in $U \times V$. But $\alpha$ is a type 1 ordinal and $\alpha \geq \beta$, so we have $\zeta \oplus \delta<\alpha$. Hence, $X \times Y$ is $\alpha$-connected im kleinen. It is easy to see that $X \times Y$ is least $\alpha$-connected im kleinen.

(2) Since $\alpha \geq 2$ is a type 2 ordinal, there exists an ordinal $\gamma<\alpha$ such that $\gamma \oplus \gamma \geq \alpha$. Since $X$ is least $\alpha$-connected im kleinen, $X$ is not $\gamma$ connected im kleinen at some point $p$ in $X$. By Corollary 12, there exists a closed neighborhood $W$ of $p$ such that, for every open neighborhood $O$ of $p, H(O, W)^{(\gamma)} \neq \varnothing$. We will show that $X \times X$ is not $\alpha$-connected im kleinen at $(p, p)$. For otherwise, there exists an open neighborhood $U$ of $(p, p)$ such that $H(U, W \times W)^{(\alpha)}=\varnothing$, by Theorem 11. Let $V$ be a closed neighborhood of $p$ contained in Int $W$ such that $V \times V \subset U$. Then $H(V \times V, W \times W)^{(\alpha)}=\varnothing$. But $H(V \times V, W \times W)=H(V, W) \times H(V, W)$ and $H(V, W)^{(\gamma)} \neq \varnothing$ by the choice of $W$. Hence, by Theorem 5 and Proposition 5, and because $\gamma \oplus \gamma \geq \alpha$, $H(V \times V, W \times W)^{(\alpha)} \neq \varnothing$, which is a contradiction.

Therefore, $X \times X$ is not $\alpha$-connected im kleinen at $(p, p)$. 


\section{The MAIN RESUlt}

Theorem 14. Let $\alpha \geq 2$ be a type 2-ordinal. If $X$ is a continuum that is least $\alpha$-connected im kleinen (hence, $X$ is not connected im kleinen at some point), then there is no continuous surjection from $X$ onto $X \times X$.

Proof. By Theorem 13, $X \times X$ is not $\alpha$-connected im kleinen at $(p, p)$. Hence, by Corollary 10, $X \times X$ cannot be a continuous image of $X$.

Example 3. If $\alpha$ is a nonzero ordinal, then $C\left(\omega^{\alpha}+1\right)$ is least $(\alpha+1)$-connected im kleinen and $\alpha+1$ is a type 2 ordinal. Hence, by Theorem 14, there is no continuous surjection from $C\left(\omega^{\alpha}+1\right)$ onto $\left[C\left(\omega^{\alpha}+1\right)\right]^{2}$.

Remark. If $\alpha \geq 2$ is an ordinal of type 1 , and $X$ is a continuum that is least $\alpha$ connected im kleinen, we cannot use the same argument, so Conjecture 1 is not completely proved. However, if $Z$ is the continuum in Example 2 ( $Z$ is least $\omega$-connected im kleinen and $\omega$ is the smallest type 1 ordinal), we can prove that there is no continuous surjection from $Z$ onto $Z \times Z$. On the contrary, suppose $f: Z \rightarrow Z \times Z$ is a continuous surjection. Then $f\left(\Omega_{n}(Z)\right) \supset \Omega_{n}(Z \times Z)$ for every natural number $n>0$. By the continuity of $f$ and the nestedness of the $\Omega_{n} s$, we have

$$
f\left(\bigcap_{n=1}^{\infty} \Omega_{n}(Z)\right) \supset \bigcap_{n=1}^{\infty} \Omega_{n}(Z \times Z) .
$$

However, $\cap \Omega_{n}(Z)$ is homeomorphic to the arc $[0,1]$, while $\cap \Omega_{n}(Z \times Z)$ is homeomorphic to $([0,1] \times Z) \cup(Z \times[0,1])$ and $([0,1] \times Z) \cup(Z \times[0,1])$ is not contained in any locally connected subcontinuum in $Z \times Z$.

It is interesting to note that if we let $X$ to be $Z \times Z$ where $Z$ is the continuum in Example 2, then $X$ is still least $\omega$-connected im kleinen by Theorem 13. But we cannot use the above method to prove the nonexistence of a continuous surjection from $X$ onto $X \times X$.

The following questions are of interest:

\section{Questions.}

(1) Is Conjecture 1 true?

(2) Is there a continuum $X$ such that it is not $\sigma$-connected im kleinen, and there does not exist a continuous surjection from $X$ onto $X \times X$ ?

(3) If $X$ is a nondegenerate indecomposable continuum, is there a continuous surjection from $X$ onto $X \times X$ ?

\section{REFERENCES}

1. David P. Bellamy, The cone over the Cantor set-continuous maps from both directions, Topology Conference Proceeding, Emory University, 1970.

2. Hidefumi Katsuura, Set functions and continuous mappings, $\mathrm{Ph}$. D. dissertation, University of Delaware, 1984.

Department of Mathematics and Computer Science, San Jose State University, San Jose, CALIFORNIA 95192-0103 